\title{
Toward Classical Models of Nucleons and Nuclei
}

\author{
David L. Selke
}

\section{ABSTRACT}

\begin{abstract}
Nuclear models can be complicated and far from first principles. Inspired by Mills' Grand Unified Theory of Classical Physics, we will present a model of the interior of nucleons that predicts their masses well considering its simplicity. Then we will apply Mills' nucleon model to the problem of computing nuclear binding energies. Because the theory is constrained to agree with classical physics including Maxwell's Equations and Newton's Laws, for instance lacking special forces that only operate in the nucleus, the possible compatible nuclear theories are strictly limited which should make finding them easier.
\end{abstract}

Keywords: classical, proton, neutron, nuclear
Published Online: June 20, 2020

ISSN: $2684-4451$

DOI : 10.24018 /ejphysics.2020.2.3.10

\section{L. Selke*}

New Model Arts, Binghamton, NY, USA.

(e-mail: dselke@hotmail.com)

*Corresponding Author

\section{INTRODUCTION}

Mills' Grand Unified Theory of Classical Physics (GUTCP) contains unprecedented calculations of measurable properties of atomic electrons and chemical bonds [1]. It goes far beyond products and reactants by giving complete charge distributions of electrons in molecules which may be used to compute the detailed intermediate states of reactions given initial conditions of orientation and motion. Here we continue the process of applying the principles of the theory to predict nuclear data. GUTCP theory describes nucleons as rotating spherical shells of charge having a certain charge distribution. We will consider an electromagnetic model for the gluons in the interior of the nucleons and compute the resulting mass. Then we will propose a single nuclear constituent based on the GUTCP nucleons' electromagnetic interactions to explain most of the known nuclear binding energy.

\section{NUCLEON MODEL}

The three gluons corresponding to the three quarks as in GUTCP are modeled as three photons that are trapped in the interior of the nucleon as in a resonator cavity. Each one is modeled as being like a plane wave except with a spherical wavefront that proceeds from the center outwards in all directions, encounters the inside surface of the nucleon and reflects losslessly back toward the center, and so on. The superposition of the outgoing and incoming waves makes a standing wave. In the fundamental mode of such a cavity, analogous to a microstrip resonator, the wavefront travels a half wavelength each time it traverses the cavity in one direction. The wavelength of the photons is the only parameter in the model, and it is constrained by existing measurements of the nucleons' sizes.

\section{NUCLEON MASS COMPUTATION}

The photon half wavelength determines the frequency according to $\mathrm{v}=\mathrm{f} \lambda$. The energy is then obtained from $\mathrm{E}=$ $\mathrm{hf}$ and the mass from $\mathrm{E}=\mathrm{mc}^{2}$. The photon frequency is $(299792458 \mathrm{~m} / \mathrm{s}) /\left(8.751 \times 10^{-16} \mathrm{~m} * 2\right.$ radii per diameter $* 2$ diameters per wavelength) or $8.56 \times 10^{22} \mathrm{~Hz}$. The energy per photon is $6.62607004 \times 10^{-34} * 8.56 \times 10^{22} \mathrm{~Hz}$ or $5.67 \times 10^{-11}$ $\mathrm{J}$. The mass is $5.67 \times 10^{-11} \mathrm{~J} /(299792458 \mathrm{~m} / \mathrm{s})^{2}$ or $6.31 \mathrm{x}$ $10^{-28} \mathrm{~kg}$ for one photon or $1.89 \times 10^{-27} \mathrm{~kg}$ for all three. This is 113 percent of the measured proton mass of about $1.67 \mathrm{x}$ $10^{-27} \mathrm{~kg}$.

\section{Measurement Status}

At some radius between the modern proton radius measurements, which disagree with each other and which are computed from indirect measurements assuming the correctness of quantum theory, and the radius given in GUTCP (the proton Compton wavelength), which cites an early measurement that agrees with it, the resonator cavity model produces the exact proton mass. If it is allowed that quantum theory may develop, even into something quite different as has often happened to established theories in the history of science, then the theory-based calculation part of experimental proton radius determinations must be questioned. An eventual change of a few percent in the accepted proton radius - and correspondingly in the resonant wavelength and mass prediction of the theory here presented - would then be quite possible.

\section{Nuclear Model}

The GUTCP nucleons are rotating nonuniform spherical shells of charge that have positive or negative charge maxima on the spherical surface (the neutron has equal 
amounts of positive and negative charge, but with a spatial distribution that gives it regions that locally have net charge). These charge maxima are not on the spin axis, nevertheless one pole is more positive than the other for both nucleons. When a proton and neutron overlap with a common center and radius, at least two effects are here proposed that determine their orientations in the deuteron. The more positive pole of each repels that of the other until each more-positive pole overlaps a more-negative pole of the other nucleon, and the energy in the interior magnetic field is minimized by rotating the axes so that the spins are anti-aligned and maximum cancellation of the magnetic fields occurs. This cancellation of the magnetic fields lowering the energy is here proposed to lower the mass of the particle according to $\mathrm{E}=\mathrm{mc}^{2}$. The nucleus is stable in part because this energy must be supplied again before separating the nucleons.

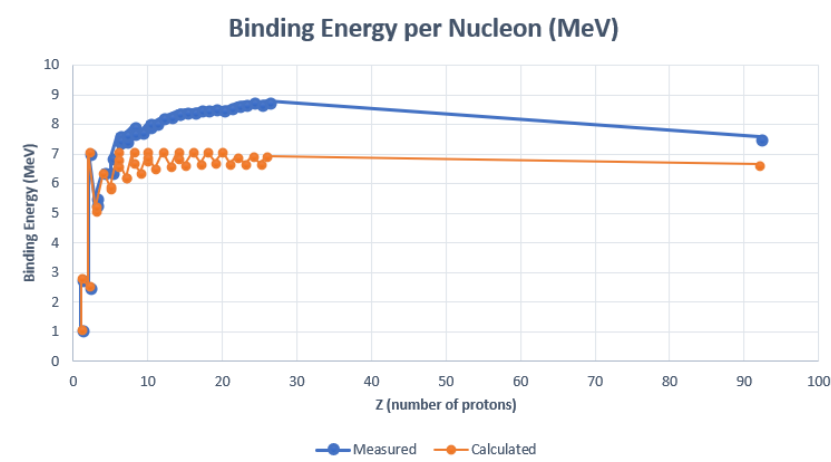

Fig. 1. Calculated vs measured binding energies of selected nuclei.

\section{HeLiUm Binding ENERGY}

The binding energies of Tritium and Helium-3 are between 3 and 4 times lower than that of Helium-4, which has only one additional nucleon. We will apply the model to explain this unusual circumstance. Like with Deuterium discussed above, the two protons of Helium- 3 and the two neutrons of Tritium anti-align so that the magnetic field of two nucleons cancels out to reduce the mass of the nucleus. In Helium-4, a pair each of protons' and neutrons' fields cancel, twice as many as in $\mathrm{T}$ and He-3. However, the energy stored in a magnetic field grows as the field squared. So there are about $2^{2}=4$ times the energy in the cancelled field (binding energy) in $\mathrm{He}-4$ as in $\mathrm{He}-3$ and $\mathrm{T}$. The somewhat lower than 4 actual ratios may be due to the neutron's field being weaker than the proton's as suggested by the neutron's smaller magnetic moment.

\section{BINDING ENERGIES OF HEAVIER ELEMENTS}

When both protons and both neutrons are spin-paired in He-4, due to the symmetry of the spin-up neutron with the spin-down neutron and the spin-up proton with the spindown proton, there is no additional magnetic field in the interior to cancel out. In the proposed model, this is why additional nucleons do not continue to quadruple the binding energy. The sharp peak at He-4 in the binding energy per nucleon graph is already close to the maximum for all elements. The "complete shell" aspect of He-4 considering both the electromagnetic forces of the constituents on each other and also the lack of additional internal field to cancel suggest that nuclear structure may consist of multiple He-4 (as many as fit) and incomplete "shells" $\mathrm{p}^{+}, \mathrm{n}^{0}, \mathrm{D}, \mathrm{T}, \mathrm{He}-3$, or dineutron. The emission of the alpha particle during decay also suggests that the alpha particle exists in the nucleus. The binding energies of several isotopes are presented in Figure 1 as the sum of the binding energies of constituent He-4 and the stated partial shells that they contain, forming as many alpha particles as possible. The dineutron was assigned the binding energy of Tritium, since both are considered to contain spin-paired neutrons that contribute the binding energy.

\section{CONCLUSION}

A simple, classical electromagnetic model was proposed for the interior of the nucleons having macroscopic analogs and no free parameters. Most of the binding energy of nuclei was explained by an approach inspired by GUTCP. Further work should aim for improved accuracy and broader scope, absolute rather than relative determination of the shell and partial shell binding energies, and should attempt an explanation of stability and decay.

\section{REFERENCES}

[1] R. Mills, The Grand Unified Theory of Classical Physics, Cranbury, NJ: Brilliant Light Power, 2020, ch. 1. 